\title{
A Ku-band wideband low noise amplifier
}

\author{
Yao Yao, Dongxu Yue, Kaiqi Wan
}

Sichuan Institute of Solid State Circuits, China Electronics Technology Group Corp., Chongqing 400060, P. R. China

Keywords: Ku-band, LNA, low noise, amplifier

Abstract: A Ku-band wideband low noise amplifier is presented in this paper. This low noise amplifier has been realized by $0.15 \mathrm{um}$ GaAs process.it exhibits high performance: over $12 \mathrm{GHz} 18 \mathrm{GHz}$, power gain is above $18.5 \mathrm{~dB}$; the ripple variation of power gain is less than $\pm 0.7 \mathrm{~dB}$; The $1 \mathrm{~dB}$ compression point is more than $12 \mathrm{dBm}$; input VSWR is lower than 1.46; output VSWR is lower than 1.55; Current less than 35mA.

\section{Introduction}

Low noise amplifiers are widely used in microwave communication, GPS receivers, remote sensing remote control, radar, electronic countermeasures, radio astronomy, geodetic mapping, television and various high precision microwave measurement systems. It is the first-stage signal processing device under the antenna. Its main function is to amplify the received weak signal to minimize the increase of noise, so as to ensure the minimum noise attenuation of the system at very low power levels. Its performance directly affects the performance of the entire receiver system, especially the sensitivity of the receiver. Therefore, the design of the low noise amplifier is the key to the design of the communication receiver.

If a high-performance low-noise amplifier is connected to the front end of the receiving system, and the noise of the latter circuit can be suppressed with a sufficiently large gain of the low-noise amplifier, the noise of the entire receiver system will mainly depend on the noise of the amplifier. The noise figure of the preamplifier has the greatest influence on the noise of the whole microwave system, and its gain will determine the degree of noise suppression for the latter stage circuit. Its linearity will have an important influence on the linearity of the whole system and the common mode noise rejection ratio. It can be seen that the performance of the microwave low noise amplifier restricts the performance of the entire receiving system, and plays a decisive role in improving the technical level of the entire receiving system. The basic requirements for low noise amplifiers are: low noise figure, adequate power gain, good operational stability, adequate bandwidth and large dynamic range. If the noise figure of the low noise amplifier is reduced, the noise figure of the receiver system is also reduced, the signal-to-noise ratio is improved, and the sensitivity is greatly improved.

\section{Source of noise}

Noise generally refers to internal noise and can be divided into natural noise and artificial noise. Natural noise includes thermal noise, shot noise, and flicker noise. Human noise includes AC noise, induced noise, and poor contact noise. In theory, any electronic circuit has electronic noise, but usually the intensity of electronic noise is very weak, mainly affecting circuits with weak signals. In electronic circuits, there are two main sources of noise: resistance noise and noise from semiconductor tubes.

\subsection{Resistance thermal noise}

When a thermal noise current flows within the resistor, a thermal noise voltage is formed. Since this voltage is random, it is not possible to characterize it with an instantaneous value, usually with a root mean square $E_{n}$. To represent. Thus, the effect of any resistor in the circuit is equivalent to the 
series connection of a resistor $\mathrm{R}$ and its thermal noise voltage $\mathrm{E}_{\mathrm{n}}$.

\subsection{Semiconductor tube noise}

\subsubsection{Diode noise}

The noise of the crystal diode is mainly the shot noise generated by the PN junction. When the diode is positively biased, it is mainly the shot noise generated when the DC passes through the PN junction. The thermal noise generated by the bulk resistance of the semiconductor material is negligible. When the reverse bias is small, the generated shot noise is also small. small. If the reverse breakdown is reached, there are two cases: the Zener breakdown diode is mainly shot noise, and the individual has 1/f noise; the avalanche breakdown diode has a large noise, except for the shot noise, the noise The voltage is randomly converted at two or more different levels, which is caused by variations in impurity defects and junction widths in the die.

\subsubsection{Bipolar transistor triode noise}

Generally, in an amplifying circuit, the noise of a transistor is often much stronger than that of a resistor. In the transistor, there are mainly two types of noise: thermal noise of the base body resistance and PN junction shot noise (generally, the shot noise is larger than the resistance thermal noise). In addition, there is still $1 / \mathrm{f}$ noise in the low frequency band.

\subsubsection{FET noise}

In the field effect transistor, since the working principle is not based on the movement of minority carriers, the influence of shot noise is small. The channel is a physical resistor that will generate thermal noise, which is the main source of noise for FETs. In addition, the channel will also converge the channel potential through the gate capacitance to the gate, resulting in induced gate noise. There is also a $1 / \mathrm{f}$ noise in the drain current. In general, the total noise of the FET is less than the noise of the transistor.

\section{Main design indicators for low noise amplifiers}

\subsection{Power gain and gain flatness}

Power gain is defined as the ratio of the real power obtained by the load to the actual power input to the network when the network input and output are arbitrary impedances.

Gain flatness refers to the fluctuation of the power gain in the operating frequency band. It is usually expressed as the difference $\Delta \mathrm{Gp}(\mathrm{dB})$ between the highest gain and the lowest gain. For low noise amplifiers, it is usually desirable to make the gain change as gentle as possible throughout the operating band. The gain is abrupt, so the smaller the $\Delta \mathrm{Gp}(\mathrm{dB})$ value, the better.

\subsection{Input and output voltage standing wave ratio}

In many cases, the characteristics of a microwave transistor amplifier are described by the input voltage standing wave ratio and the output voltage standing wave ratio. In low noise amplifiers, the input and output voltage standing wave ratios characterize the matching of their input and output loops.

In designing the matching circuit of the low noise amplifier, the input matching network is generally designed as the optimal noise matching network instead of the optimal power matching network to obtain the minimum noise, and the output matching network is generally used to obtain the maximum power and the lowest standing wave ratio. design. Therefore, there is always some kind of mismatch at the input of the low noise amplifier. 


\subsection{Stability}

The stability of the RF amplifying circuit refers to the ability of the amplifying circuit to suppress environmental changes (such as temperature changes, frequency drift, signal source impedance, and load impedance characteristics) to maintain normal amplification characteristics. The stability of the RF amplifying circuit is a very important factor in maintaining the normal operation of the communication system.

In the stability analysis of the RF amplifying circuit, the amplifying circuit is usually analyzed as a two-port network with known S parameters.

Due to the reverse voltage transmission characteristic of the transistor, the voltage reflection coefficient TL of the load terminal can be reflected at the input port of the amplifier through the reverse voltage transmission coefficient $S_{12}$ of the transistor, that is, there is a certain relationship between $\Gamma_{\mathrm{IN}}$ and $\Gamma_{\mathrm{L}}$. After the input RF signal passes through the forward amplification, load reflection and reverse transmission of the transistor, it is reflected as a reflected signal at the input end, and its amplitude may be larger than the input signal amplitude, resulting in instability of the amplification circuit. Similarly, the reflection coefficients $\Gamma_{\text {OUT }}$ and $\Gamma$ s at the output of the amplifier circuit have a similar relationship. Therefore, the stability of the amplifier circuit is not only related to the s-parameter, but also related to the input and output matching network of the amplifying circuit, the source impedance and the load impedance. In addition, since the S-parameter of the transistor two-port network depends on the operating frequency, the RF transistor amplifying circuit can work normally at certain frequencies, and at other frequencies, it cannot work normally. Therefore, the stability of the circuit is also related to the operating frequency.

\section{Circuit structure selection}

Considering that if a first-stage amplifier is used to complete the bandwidth requirement with a tube, the circuit form should use a negative feedback type circuit; however, the disadvantage of the negative feedback type amplifier is that the noise figure is high, which makes it difficult to meet the low noise requirement, and The gain target is also difficult to achieve, so it is more difficult to use a first-order amplifier. Designed as a two-stage amplifier, the noise figure analysis of the cascaded network shows that the noise figure is mainly determined by the first-stage circuit in the two-stage amplifier circuit, so that in the circuit design, the first-stage amplifier circuit should focus on noise reduction. The design, while the second stage amplifying circuit focuses on the design of the boost gain.

The specific requirements of the matching circuit are: input matching is low noise matching, and has better input standing wave; inter-stage matching requires low noise and maintains flat gain; output matching is high gain matching, gain is flat and output standing wave is good .

Since the design is Ku-band, its $1 / 4$ wavelength line is about $1 \mathrm{~cm}$, and the size is still very large. It is not suitable for use in MMIC, so it is not considered to use too many microstrip lines for matching, and the lumped component is in $\mathrm{X}$. The band has a relatively good model to characterize, so consider using lumped element resistance, inductance, and capacitance to match. Since the HEMT device used in this design has a high single-tube gain, the two-stage amplifier can basically achieve the gain requirement. In the function definition of the two levels of the circuit, the first stage has the requirement of low noise as the maximum, while taking into account the gain and input matching, while the second stage mainly solves the gain and the output standing wave ratio. The lumped components are connected between the two stages to reduce losses and improve the stability of the circuit. The first and second stages use the same structure.

The circuit structure selected for this circuit is shown in the figure 1 


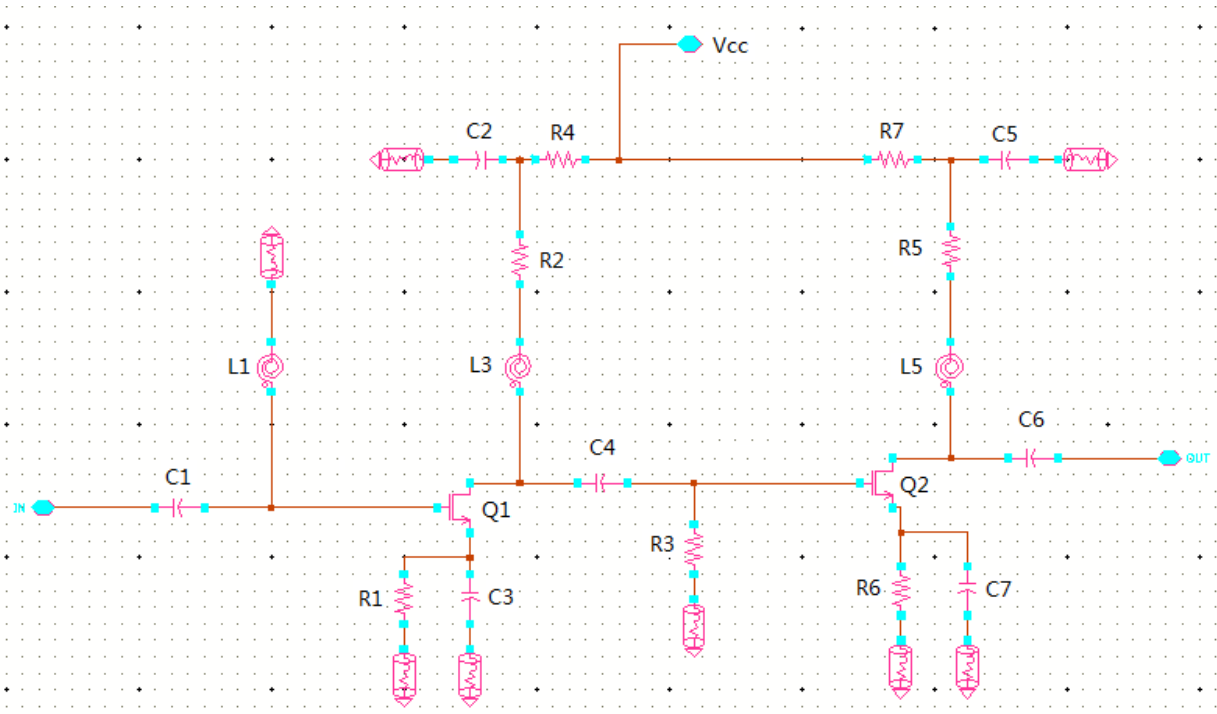

Figure 1 Circuit structure

\section{Circuit design}

Using the suitable configuration, a Ku-band wideband low noise amplifier has been realized by 0.15um GaAs process. The simulation of our LNA have been presented based on the ADS2015. Over $12 \mathrm{GHz} 18 \mathrm{GHz}$, power gain is above $18.5 \mathrm{~dB}$ (Figure 2); the ripple variation of power gain is less than $\pm 0.7 \mathrm{~dB}$; The noise figure is less than $1.5 \mathrm{~dB}$ (Figure 3 ); The $1 \mathrm{~dB}$ compression point is more than $12 \mathrm{dBm}$ (Figure 4); input VSWR is lower than 1.46 (Figure 5); output VSWR is lower than 1.55 (Figure 6); Current less than 35mA. the layout of the LNA with a chip size of $1.0 * 1.35 \mathrm{~mm}^{2}$.

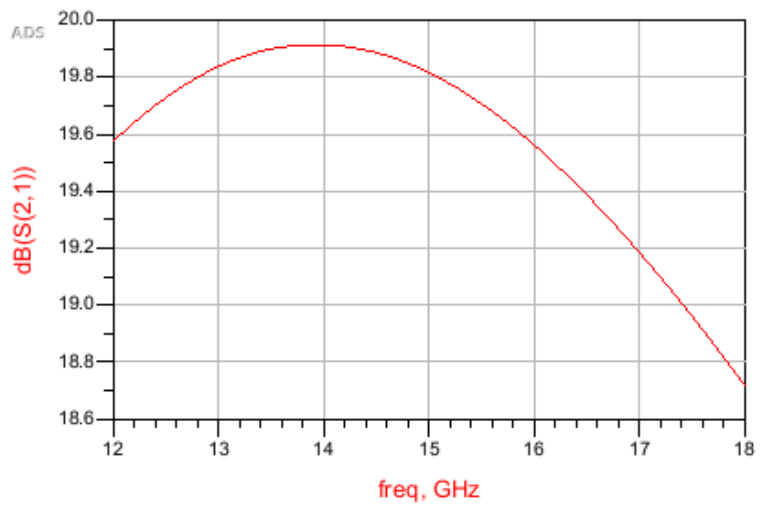

Figure 2 Simulated Power Gain

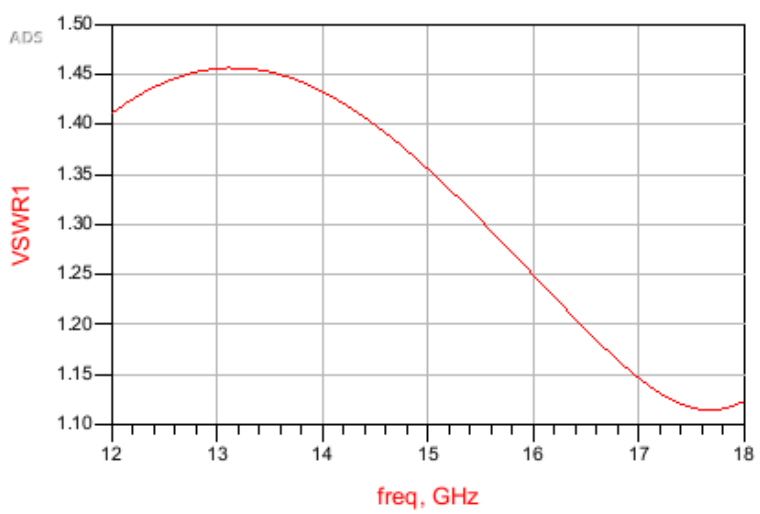

Figure 4 Simulated input VSWR

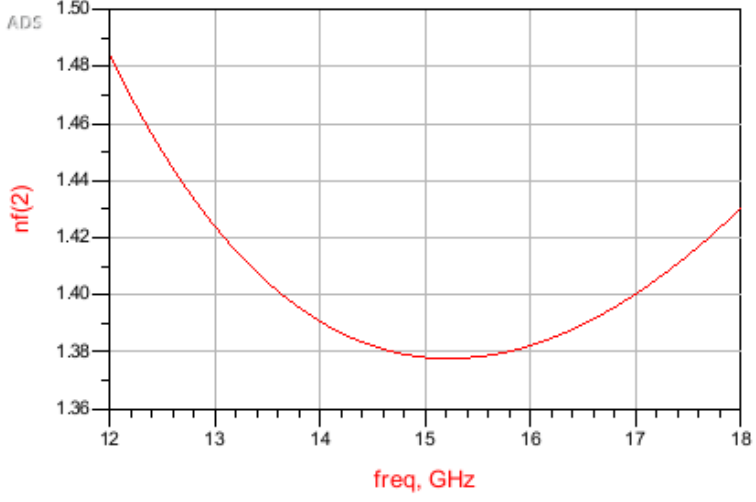

Figure3 Simulated Noise figure

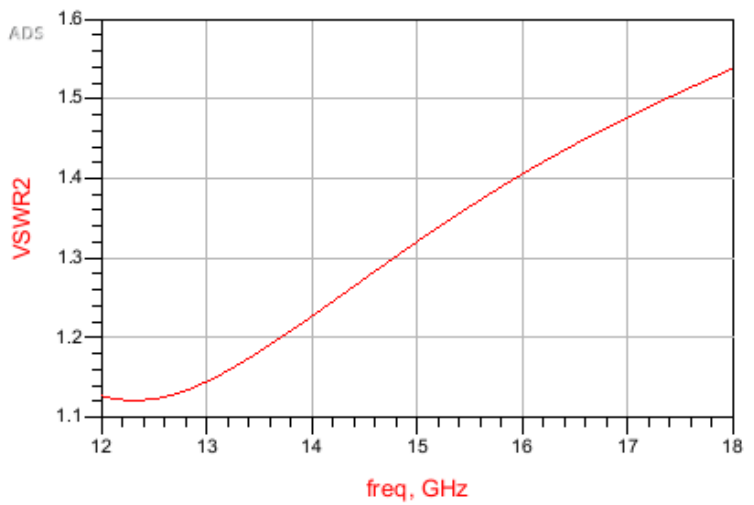

Figure 5 Simulated ouput VSWR 


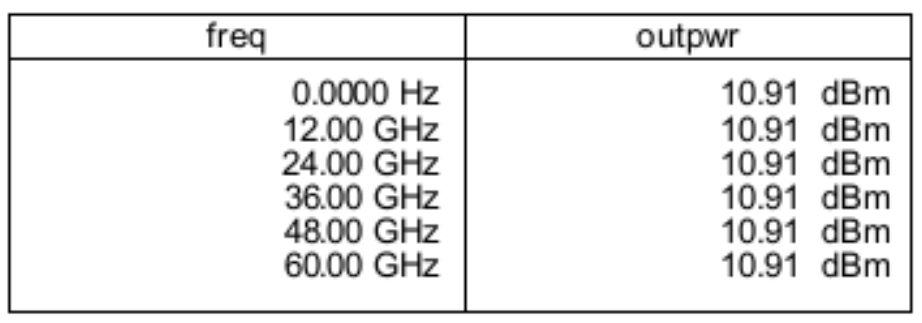

Figure 6 Simulated Output $1 \mathrm{~dB}$ compression point

\section{Conclusions}

In this paper a suitable configuration is used for a Ku-band wideband low noise amplifier. the simulation results shows over $12 \mathrm{GHz} 18 \mathrm{GHz}$, power gain is above $18.5 \mathrm{~dB}$; the ripple variation of power gain is less than $\pm 0.7 \mathrm{~dB}$; The noise figure is less than $1.5 \mathrm{~dB}$; The $1 \mathrm{~dB}$ compression point is more than 12dBm; input VSWR is lower than 1.46; output VSWR is lower than 1.55; Current less than $35 \mathrm{~mA}$.. the proposed one is good candidate for Ku-band microwave monolithic low-noise amplifer applications.

\section{References}

[1] Edward C. Niehenke, Robert A, Pucel, Inder J. Bahl. Microwave and Millimeter-Wave Integrated Circuits. IEEE Transactions on Microwave Theory and Techniques, March 2002, 50(3): 846-848.

[2] S. Cha, Y.H. Chung, m. Wojtowwicz, et al. "Wideband A1GaN/GaN HEMT MMIC Low Noise Amplifier for Highly Microwave Symposium Digest, IEEE MTT-S Survivable Receiver Electronics" [J],International, 2004, pp.829-832.

[3] Pengelly R. S., Turner J.A. Monolithic Broad GaAs FET Amplifier" [J] Electronics Letters, 1976, Page(s): 251-252.

[4] P.J.Meier, P.R.Bie, J. DeCarlo. Integrated MMIC and MIC components for multichannel Ka-band receivers. Microwave Symposium Digest, 1992. IEEE MTT-S International, June 1992: 1099-1100.

[5] William R. Deal, Michael Biedenbender, et al. "Design and Analysis of Broadband Dual-Gate Balanced Low-Noise Amplifier" [J], IEEE Journal of Solid-State Circuits, Vo1.42, No.42, No. 10, Oct. 2007, Pa\}e(s):2107-2115.

[6] Zhang Zongnan, Huang Qinghua, Hao Mingli, et al. "Design of a 24-40 GHz balanced low noise amplifier using Lange couplers"[J], Journal of Semiconductors,Vo1.30, No. 4, April 2009.

[7] Parikh P, Wu Y, Moore M,LNA\&receiver ICs" [J],et al. "High linearity, robust, A1GaN-GaN HEMTs for High Performance Devices 2002 Proceedings, IEEE Lester Eastman Conference on 6-8 Aug. 2002, Pages(s): 415-421.

[8] A.K.Ezzeddine, H.L.A.Hung. A novel power combiner for MIC and MMIC amplifiers. Microwave Symposium Digest, 1990. IEEE MTT-S International, May 1990: 479-482.

[9] Mingqi Chen, William Sutton, et al. "A 1-25 GHz GaN HEMT MMIC Low Noise Amplifier" [J], IEEE Microwave and Wireless Components Letters, Vo1.20, No. 10, October 2010, Page(s):563-565.

[10] S. Cha, Y.H. Chung, M. Wojtowicz, I. Smorchkova, B.R. Allen, J.M. Yang, R.Kagiwada. "Wideband AlGaN/GaN HEMT Low Noise Amplifier for Highly Survivable Receiver Electronics" [C], IEEE MTT-S International. Microwave Symposium Digest, 2004, page(s): 829-831.

[11] J.James, Whelehan. Low Noise Amplifiers-Then and Now [J]. IEEE Transactions on Microwave 
Theory Tech. March 2002, 50(3): 806-813.

[12] M. Micovic, A. Kurdoghlian, H. P. Moyer, et al. "GaN MMIC Technology for Microwave and Millimeter-wave Applications" [J], IEEE Semiconductor Integrated Circuit Symposium, 30 Oct.-2 Nov. 2005, Page(s):173-176.

[13] Parikh P, Wu Y, Moore M, et al. "High linearity, robust, A1GaN-GaN HEMTs for LNA\&receiver ICs" [J], High Performance Devices 2002 Proceedings, IEEE Lester Eastman Conference on 6-8 Aug. 2002, Page(s):415-421 